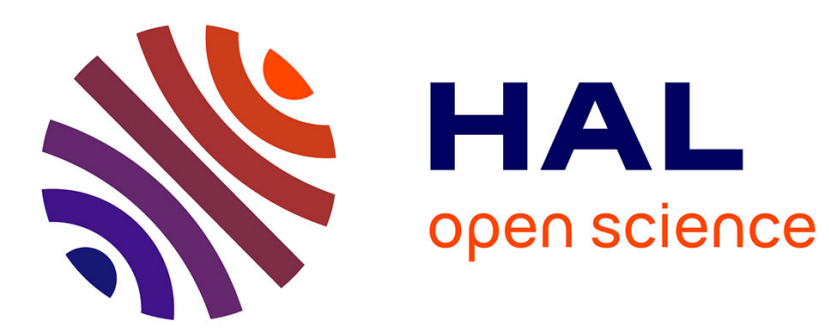

\title{
A Schur algorithm for symmetric inner functions
}

Martine Olivi, Bernard Hanzon, Ralf L.M. Peeters

\section{To cite this version:}

Martine Olivi, Bernard Hanzon, Ralf L.M. Peeters. A Schur algorithm for symmetric inner functions.

Conference on Decision and Control, Dec 2005, Seville, Spain. hal-00753556

\section{HAL Id: hal-00753556 \\ https://hal.inria.fr/hal-00753556}

Submitted on 19 Nov 2012

HAL is a multi-disciplinary open access archive for the deposit and dissemination of scientific research documents, whether they are published or not. The documents may come from teaching and research institutions in France or abroad, or from public or private research centers.
L'archive ouverte pluridisciplinaire HAL, est destinée au dépôt et à la diffusion de documents scientifiques de niveau recherche, publiés ou non, émanant des établissements d'enseignement et de recherche français ou étrangers, des laboratoires publics ou privés. 


\title{
A Schur algorithm for symmetric inner functions.
}

\author{
Martine Olivi, Bernard Hanzon, Ralf L.M. Peeters
}

\begin{abstract}
Symmetric inner rational functions naturally arise in the description of physical systems which satisfy the conservation and reciprocity laws. Inner matrix functions can be parametrized by a sequence of interpolation vectors obtained from a tangential Schur algorithm. In this paper, we present a Schur type algorithm which allows to describe symmetric inner functions, based on a two-sided Nudelman interpolation problem. This Schur algorithm gives rise to an interesting interpretation in the context of surface acoustic wave filters.
\end{abstract}

\section{INTRODUCTION.}

Symmetric inner functions naturally arise in the description of physical systems which satisfy the conservation and reciprocity laws. This study was initially motivated by an application to the synthesis of SAW (surface acoustic wave) filters. A SAW filter can be viewed as composed by a finite number of cells, each cell containing a reflection center with reflection coefficient $\left.r_{i}, r_{i} \in\right]-1,1[$, and an electroacoustic center [3]. The acoustic matrix which relates ingoing waves to outgoing waves can be computed from the sequence of reflection coefficients $r_{1}, r_{2}, \ldots, r_{N}$. Put

$$
t_{n}=\sqrt{1-r_{n}^{2}}, \quad P_{n}=t_{1} t_{2} \ldots t_{n}
$$

and define Schur polynomials $\phi_{n}$ and $\psi_{n}$ of degree $(n-1)$ satisfying the Levinson recursions

$$
\left\{\begin{array}{l}
\phi_{n}(z)=z \phi_{n-1}(z)+r_{n} \psi_{n-1}(z) \\
\psi_{n}(z)=z r_{n} \phi_{n-1}(z)+\psi_{n-1}(z)
\end{array}\right.
$$

with

$$
\left\{\begin{array}{l}
\phi_{1}(z)=1 \\
\psi_{1}(z)=r_{1}
\end{array}\right.
$$

Note that these polynomials have real coefficients. Also define the reciprocal polynomial of a polynomial $p(z)$ of formal degree $n$ as

$$
\tilde{p}(z)=z^{n} \bar{p}(1 / z)=z^{n} \overline{p(1 / \bar{z})} .
$$

The acoustic matrix associated with the first $n$-cells of the filter is thus given by

$$
Q_{n}(z)=z\left[\begin{array}{cc}
-j \psi_{n}\left(z^{2}\right) & P_{n} z^{n-1} \\
P_{n} z^{n-1} & -j \widetilde{\psi}_{n}\left(z^{2}\right)
\end{array}\right] / \widetilde{\phi}_{n}\left(z^{2}\right)
$$

INRIA, BP 93, 06902 Sophia-Antipolis Cedex, FRANCE, \{olivi\}@ sophia.inria.fr, phone: 33-4-92387877, fax: 33-4-92387858

School of Mathematical Sciences, University College, Cork, Ireland, Tel.: +353-21-4902376, Fax +353-21-4903784, E-mail: b.hanzon@ucc.ie

Dept. Mathematics, Universiteit Maastricht, P.O. Box 616, 6200 MD Maastricht, The Netherlands, Tel.: +31-43-3883365, Fax: +31-43-3884910, Email: ralf.peeters@math.unimaas.nl in which $j$ denotes the square root of -1 . Note that, $\widetilde{\phi}_{n}\left(z^{2}\right)=z^{2(n-1)} \phi_{n}\left(1 / z^{2}\right), \widetilde{\psi}_{n}\left(z^{2}\right)=z^{2(n-1)} \psi_{n}\left(1 / z^{2}\right)$. The matrices $Q_{n}(z)$ are symmetric inner matrices, of McMillan degree $2 n$, with complex coefficients. In this example, the complex structure is very particular, but it can be much more complicated if one considers a combination of filters. Rational complex matrices which are both inner and symmetric seem to be relevant objects in physics and electrical engineering. However, while there exists an important literature on inner functions and some papers on real symmetric rational matrix functions [6], [7], to our knowledge symmetric inner matrices have not been studied.

Tangential Schur algorithms provide interesting tools to parametrize inner functions by means of interpolation values [1], [5], [10]. The object of this paper is to present a Schur algorithm which allows to describe symmetric inner functions. In order to take into account symmetry, the usual NevanlinnaPick interpolation problem underlying the Schur algorithm will be replaced by a two-sided Nudelman interpolation problem.

\section{SCHUR ALGORITHMS, INTERPOLATION AND PARAMETRIZATION.}

The Schur algorithm was originally a nice recursive test for checking the boundedness of an analytic function $S(z)$ in the disk: define a sequence of functions by $S_{0}=S$ and

$$
S_{i+1}(z)=\frac{S_{i}(z)-S_{i}(0)}{z\left(1-S_{i}(0)^{*} S_{i}(z)\right)}
$$

then, $|S(z)|<1$ for $|z|<1$ if and only if $\left|S_{i}(0)\right|<1$ for all $i \geq 0$. Such an analytic function is called a Schur function and it is completely characterize by the sequence of numbers $\gamma_{i}=S_{i}(0)$. Then, Schur type algorithms were used to approach interpolation problems with metric constraints (Nevanlinna-Pick problems): find a Schur function which takes certain specified values at certain points inside the unit disk.

Generalizations of Nevanlinna-Pick problems to the matrix case were considered, often motivated by some sample problems in control and system theory: sensitivity minimization, robust stabilization and model reduction. For these questions we refer the reader to [2] and the bibliography therein. An interpolation condition for a rational matrix function can take 
several forms, namely

$$
\begin{aligned}
S(w) u & =v, \\
x^{T} S(w) & =y^{T}, \\
x^{T} S(w) u & =\rho, \\
S(w) & =M,
\end{aligned}
$$

in which $w$ is a complex scalar, $u, v, x, y$, are vectors and $M$ is a complex matrix. The solutions of such interpolation problems can be usually parametrized via a linear fractional transformation, that is a transformation of the form

$$
T_{\Theta}(R)=\left(\Theta_{1} R+\Theta_{2}\right)\left(\Theta_{3} R+\Theta_{4}\right)^{-1},
$$

associated with some rational matrix function $\Theta(z)$ with block decomposition

$$
\Theta=\left[\begin{array}{ll}
\Theta_{1} & \Theta_{2} \\
\Theta_{3} & \Theta_{4}
\end{array}\right] .
$$

The most popular interpolation problem, also called Nevanlinna-Pick problem consists of a set of directional interpolation conditions of the form (3). Schur algorithms to solve this problem were presented among others in [1], [10]. In [1] a Schur algorithm is used to provide parameters for the set of rational inner functions of fixed McMillan degree. We briefly recall this result.

Let

$$
J=\left[\begin{array}{cc}
I_{p} & 0 \\
0 & -I_{p}
\end{array}\right], \quad K=\left[\begin{array}{cc}
0 & I_{p} \\
I_{p} & 0
\end{array}\right] .
$$

For any matrix function $F(z)$, we define

$$
F^{\sharp}(z)=F(\bar{z})^{*}, \quad \bar{F}(z)=\overline{F(\bar{z})} .
$$

A $2 p \times 2 p$ rational matrix function $\Theta(z)$ is called $J$-inner if, at every point of analyticity $z$ of $\Theta$ it satisfies

$$
\begin{aligned}
& \Theta(z) J \Theta(z)^{*} \leq J, \quad|z|<1, \\
& \Theta(z) J \Theta(z)^{*}=J, \quad|z|=1 .
\end{aligned}
$$

The linear fractional transformation (7) possesses the following useful properties: if $Q=T_{\Theta}(R)$ where $R$ and $Q$ are invertible, then

$$
\begin{aligned}
Q^{-1} & =T_{K \Theta K}\left(R^{-1}\right), \\
Q^{T} & =T_{K \bar{\Theta}(1 / z) K}\left(R^{T}\right) .
\end{aligned}
$$

A $p \times p$ rational matrix function $S(z)$ is Schur if it is analytic and contractive in the disk

$$
S(z) S(z)^{*} \leq I_{p}, \quad|z|<1,
$$

and inner if in addition it takes unitary values on the circle

$$
S(z) S(z)^{*}=I_{p}, \quad|z|=1 .
$$

A linear fractional transformation (7) associated with a $J$ inner matrix function $\Theta$ transforms inner functions into inner functions.

Let $Q(z)$ be a $p \times p$ inner function of McMillan degree $n$, then (i) $w \in \mathbb{C},|w|<1$ being given, we can always find some direction $u \in \mathbb{C}^{p},\|u\|=1$ such that $v$ given by the interpolation condition

$$
Q(w) u=v,
$$

has norm strictly less than 1.

(ii) in that case $(\|v\|<1)$, a $J$-inner matrix function can be built from the interpolation data $w, u, v$, namely

$$
\Theta_{w, u, v}(z)=I_{2 p}+(z-1) \frac{1-|w|^{2}}{1-\|v\|^{2}} \frac{\left[\begin{array}{c}
v \\
u
\end{array}\right]\left[\begin{array}{l}
v \\
u
\end{array}\right]^{*}}{(z-w)(1-\bar{w})} J
$$

such that the function $Q(z)$ can be represented by the linear fractional transformation (7) associated with $\Theta_{w, u, v}$, for some inner function $Q_{n-1}(z)$ of degree $(n-1)$.

The tangential Schur algorithm consists of repeating this process, and thus provides a sequence of inner functions,

$$
Q_{n}=Q, Q_{n-1}, \ldots, Q_{1}, Q_{0},
$$

in which for $k=n, n-1, \ldots, 1, Q_{k}$ has degree $k$ and satisfies the interpolation condition

$$
Q_{k}\left(w_{k}\right) u_{k}=v_{k}, \quad\left\|v_{k}\right\|<1,
$$

and $Q_{0}$ is a constant unitary matrix.

A local parametrization (or chart) for the set of inner functions of McMillan degree $n$ can be associated with a sequence of interpolation points $\left(w_{1}, w_{2}, \ldots, w_{n}\right)$ and interpolation directions $\left(u_{1}, u_{2}, \ldots, u_{n}\right)$. An inner function of McMillan degree $n$ can be parametrized in this chart, if the Schur algorithm can be run until $Q_{0}$ (i.e. for $k=$ $n, \ldots, 1$, the interpolation values $v_{k}=Q_{k}\left(w_{k}\right) u_{k}$ satisfy $\left\|v_{k}\right\|<1$ ). Note that, by (i), for any $Q(z)$, we can find a sequence of interpolation values and interpolation directions such that this condition is satisfied. The interpolation values $v_{n}=v, v_{n-1}, \ldots, v_{1}$, are then the parameters of $Q$ in the chart, together with the unitary matrix $Q_{0}$.

Recall that a linear fractional transformation $T_{H}$ associated with such a $J$-unitary matrix $H$ is a bijection on the set of inner functions which preserves the McMillan degree [8]. Moreover, if $\Theta$ is a $J$-inner function, we have that

$$
T_{\Theta H}(R)=T_{\Theta}\left(T_{H}(R)\right) .
$$

This leads to the following remark.

Remark 1: The function $\Theta_{w, u, v}$ satisfies the condition $\Theta_{w, u, v}(1)=I_{2 p}$. This implies that the constant unitary matrix $Q_{0}$ in the Schur algorithm is such that $Q_{0}=Q(1)$. This choice naturally arises in a function space approach [4]. The matrix $\Theta_{w, u, v}$ also has the nice factorization (obtained from [8, Lemma 5.1] applied to $K \Theta_{w, u, v}^{\sharp} K$ )

$$
\begin{aligned}
& \Theta_{w, u, v}(z) \\
& =H\left(v u^{*}\right)^{-1}\left[\begin{array}{cc}
I_{p} & 0 \\
0 & I_{p}+\left(\beta_{w}^{\sharp}(z)-1\right) u u^{*}
\end{array}\right] H\left(v u^{*}\right),
\end{aligned}
$$

in which $\beta_{w}$ and $H\left(v u^{*}\right)$ are defined by (18) and (21) respectively. However, assertion (ii) is still true if we replace $\Theta_{w, u, v}$ 
in the linear fractional transformation by any $\Theta_{w, u, v, H}=$ $\Theta_{w, u, v} H$, where $H$ is a constant $J$-unitary matrix. This matrix $H$ may depend (smoothly) on the interpolation data. The corresponding Schur algorithm will provide from $Q$ a different sequence of inner matrices $Q_{k}$. Such a choice may be more convenient in some context. In [10], a similar Schur algorithm is presented which gives rise to a circuit theoretical interpretation. It is described in the case of continuous-time transfer functions, but it corresponds in our discrete-time setting to the choice of $\Theta_{w, u, v} H\left(v u^{*}\right)^{-1}$. In [8], the freedom in the choice of the matrix $H$ has been used to associate with the Schur algorithm a nice recursive construction of balanced realizations. This is possible with the $J$-inner matrix

$\hat{\Theta}_{w, u, v}=H\left(w v u^{*}\right)\left[\begin{array}{cc}I_{p} & 0 \\ 0 & I_{p}+\left(\frac{1-\bar{w} z}{z-w}-1\right) u u^{*}\end{array}\right] H\left(v u^{*}\right)$.

Now, we turn to the case of symmetric functions. In view of (13) we have the following preliminary result:

Lemma 1: Let $\Theta(z)$ be a $J$-unitary function such that

$$
\bar{\Theta}(1 / z)=K \Theta(z) K \text {. }
$$

Then, the linear fractional transformation $T_{\Theta}$ preserves symmetry.

\section{ELEMENTARY SYMMETRIC INNER FUNCTIONS.}

The Schur algorithm we shall describe in the next section produces a sequence of symmetric inner functions whose McMillan degree decreases by 2 at each step. Thus, it ends either on a symmetric unitary matrix or on an inner function of McMillan degree 1. In this section, we shall characterize these elementary symmetric inner functions. As mentioned previously, constant $J$-unitary functions also play an important role in these questions. We shall also characterize $J$-unitary matrices $H$ which satisfies the condition $\bar{H}=K H K$, so that $T_{H}$ preserves symmetry.

Symmetric complex matrices have been studied in [9]. We recall the following result.

Lemma 2: A complex matrix $X$ is both symmetric and unitary if and only if it can be written as $X=$ $O \Lambda O^{T}$ where $O$ is a real orthogonal matrix and $\Lambda=$ $\operatorname{diag}\left(\lambda_{1}, \lambda_{2}, \ldots, \lambda_{n}\right), \quad\left|\lambda_{k}\right|=1$.

Every inner function of McMillan degree 1 can be written in the form (see [4, th.1.4.])

$$
B(z)=\left(I_{p}+\left(\beta_{\zeta}(z)-1\right) x x^{*}\right) X,
$$

where $\beta_{\zeta}(z)$ is the Blaschke factor

$$
\beta_{\zeta}(z)=\frac{(z-\zeta)(1-\bar{\zeta})}{(1-\bar{\zeta} z)(1-\zeta)}, \quad|\zeta|<1
$$

$X$ is a unitary matrix uniquely determined by $X=B(1)$ and $x^{*}$ is a left kernel vector of $B(\zeta)$ such that $\|x\|=1$.

If $B(z)$ is symmetric, then the matrix $X=B(1)$ is symmetric too. Since it is unitary, there exists a unitary matrix $Y$ such that (see Lemma 2)

$$
X=Y Y^{T} \text {. }
$$

Consider the matrix

$$
\hat{B}(z)=Y^{*} B(z) \bar{Y}=I_{p}+\left(\beta_{\zeta}(z)-1\right) y y^{*},
$$

where $y=Y^{*} x$. The matrix $\hat{B}(z)$ will be symmetric if and only if $\epsilon y$ is real, for some unit complex number $\epsilon$.

Lemma 3: The inner function $B(z)$ given by (17) is symmetric if and only if it can be written in the form

$$
B(z)=Y\left(I_{p}+\left(\beta_{\zeta}(z)-1\right) y y^{T}\right) Y^{T},
$$

in which $y$ is a unit real $p$-vector and $Y$ a unitary matrix.

It is known [4, Th.1.2] that every $2 p \times 2 p J$-unitary matrix can be expressed in the form

$$
H=H(E)\left[\begin{array}{ll}
P & 0 \\
0 & Q
\end{array}\right]
$$

where $P$ and $Q$ are $p \times p$ unitary matrices, and $H(E)$ the Halmos extension of a strictly contractive $p \times p$ matrix $E$, that is

$$
H(E)=\left[\begin{array}{cc}
\left(I_{p}-E E^{*}\right)^{-1 / 2} & E\left(I_{p}-E^{*} E\right)^{-1 / 2} \\
E^{*}\left(I_{p}-E E^{*}\right)^{-1 / 2} & \left(I_{p}-E^{*} E\right)^{-1 / 2}
\end{array}\right] .
$$

Lemma 4: The J-unitary matrix $H(E)$ satisfies $\overline{H(E)}=K H(E) K$ if and only if $E$ is symmetric.

\section{A SCHUR ALGORITHM FOR SYMMETRIC INNER FUNCTIONS.}

Let $Q$ be a $p \times p$ symmetric inner function of McMillan degree $n \geq 2$ and assume it satisfies the Nevanlinna-Pick interpolation condition

$$
Q(w) u=v
$$

where $w$ belongs to the open unit disk, $u$ and $v$ are complex $p$-vectors, $\|u\|=1$ and $\|v\|<1$. In order to take into account the symmetry of $Q(z)$, we shall consider simultaneously the symmetric interpolation condition

$$
u^{T} Q(w)=v^{T} .
$$

The interpolation problem: find $Q$ which satisfies (22) and (23), is a special case of the two-sided Nevanlinna-Pick problem in which the interpolation points coincide. It can be viewed as a two-sided Carathéodory-Fejér problem [4, th.6.3.] or as a particular case of a the two-sided Nudelman interpolation problem considered in [2, ex.18.5.3]. For this problem to be well-posed, an extra interpolation condition of the form

$$
u^{T} Q^{\prime}(w) u=\rho,
$$

in which $Q^{\prime}$ denotes the derivative of $Q$, must be added to the previous ones.

Put

$$
\sigma=\frac{1-\|v\|^{2}}{1-|w|^{2}}
$$

and associate with the set of interpolation data

$$
\delta=(w, u, v, \rho),
$$


the Pick matrix

$$
\Lambda_{\delta}=\left[\begin{array}{ll}
\sigma & \bar{\rho} \\
\rho & \sigma
\end{array}\right]
$$

and the $J$-inner matrix function

$$
\begin{aligned}
\Theta_{\delta}(z)=I_{2 p}+\quad & (z-1) C\left[\begin{array}{cc}
(z-w)^{-1} & 0 \\
0 & (1-z \bar{w})^{-1}
\end{array}\right] \\
& \Lambda_{\delta}^{-1}\left[\begin{array}{cc}
(1-\bar{w})^{-1} & 0 \\
0 & (1-w)^{-1}
\end{array}\right] C^{*} J
\end{aligned}
$$

with

$$
C=\left[\begin{array}{ll}
v & -\bar{u} \\
u & -\bar{v}
\end{array}\right]
$$

Remark 2: Note that $\Theta_{\delta}(1)=I_{2 p}$ and that $\bar{\Theta}_{\delta}(1 / z)=$ $K \Theta_{\delta}(z) K$, so that by Lemma 1 the linear fractional transformation $T_{\Theta_{\delta}}$ preserves symmetry.

It is proved in [2] that there exists a Schur function satisfying the interpolation conditions (22), (23) and (24) if and only if the Pick matrix $\Lambda_{\delta}$ is positive definite. In the case of a symmetric inner function we have the following representation theorem.

Proposition 1: Let $Q(z)$ be a $p \times p$ symmetric inner function of McMillan degree $n \geq 2$ which satisfies (22), (23) and (24) and such that $\Lambda_{\delta}$ is positive definite. Then, $Q(z)$ can be represented as

$$
Q=T_{\Theta_{\delta}}(R),
$$

where $\Theta_{\delta}$ is given by (28) and $R$ is a $p \times p$ symmetric inner function of McMillan degree $(n-2)$.

Proof. We give a constructive proof of this result which follows the approach developed by Potapov to describe the multiplicative structure of $J$-inner functions [11]. We first consider the case where the interpolation data are of the form $\delta_{0}=(0, u, 0, \rho)$, so that (22) takes the simpler form

$$
Q(0) u=0 .
$$

From $\delta_{0}$ we construct the positive definite matrix $\Lambda_{\delta_{0}}$ given by

$$
\Lambda_{\delta_{0}}=\left[\begin{array}{cc}
1 & \bar{\rho} \\
\rho & 1
\end{array}\right], \quad|\rho|<1
$$

and the matrix $\Theta_{\delta_{0}}(z)$ can be easily computed as

$$
\Theta_{\delta_{0}}(z)=\left[\begin{array}{cc}
I_{p}+\frac{z-1}{1-|\rho|^{2}} \bar{u} u^{T} & -\frac{\rho(z-1)}{1-|\rho|^{2}} \bar{u} u^{*} \\
\frac{\bar{\rho}(z-1)}{\left(1-|\rho|^{2}\right) z} u u^{T} & I_{p}-\frac{z-1}{\left(1-|\rho|^{2}\right) z} u u^{*}
\end{array}\right] .
$$

Then, we write the Taylor series of $Q(z)$ about 0 :

$$
Q(z)=Q(0)+z Q^{\prime}(0)+\ldots
$$

and the singular value decomposition of $Q(0)$

$Q(0)=V \operatorname{diag}\left(0, \ldots, 0, \lambda_{1}, \ldots, \lambda_{r}\right) U^{*}, 0<\lambda_{1} \leq \ldots \leq \lambda_{r}$,

for some unitary matrices $U$ and $V$. From (22) we may choose the vector $u$ as first column vector of $U$. Now, let

$$
B(z)=I_{p}+(z-1) u u^{*}=U \operatorname{diag}(z, 1, \ldots, 1) U^{*},
$$

then $Q(z)=Q_{1}(z) B(z)$ for some inner function $Q_{1}(z)$ of McMillan degree $(n-1)$, and

$Q_{1}(z)=Q(z) B(z)^{-1}=Q^{\prime}(0)\left(z I_{p}+(1-z) u u^{*}\right)+z \times \ldots$

We then have the following interpolation condition for $Q_{1}(z)$

$$
u^{T} Q_{1}(0)=u^{T} Q^{\prime}(0) u u^{*}=\rho u^{*} .
$$

This interpolation condition differs from (3) but can be approached in a similar way [5]. Since $\|\rho u\|=|\rho|<1$, its solutions can be represented by a linear fractional transformation $Q_{1}=T_{\Theta_{1}}\left(R_{1}\right)$ for some inner matrix function $R_{1}$ of McMillan degree $(n-2)$ (since $Q_{1}$ has McMillan degree $(n-1))$, and where $\Theta_{1}$ is the $J$-inner function

$$
\Theta_{1}(z)=I_{2 p}+(z-1) \frac{\left[\begin{array}{c}
\bar{u} \\
\bar{\rho} u
\end{array}\right]\left[\begin{array}{c}
\bar{u} \\
\bar{\rho} u
\end{array}\right]^{*} J}{1-|\rho|^{2}} .
$$

Thus

$$
\begin{aligned}
Q_{1}(z)= & \left(\left(I_{p}+\frac{(z-1)}{\left(1-|\rho|^{2}\right)} \bar{u} \bar{u}^{*}\right) R_{1}(z)-\frac{(z-1)}{\left(1-|\rho|^{2}\right)} \rho \bar{u} u^{*}\right) \\
& \left(\frac{(z-1)}{\left(1-|\rho|^{2}\right)} \bar{\rho} u \bar{u}^{*} R_{1}(z)+I_{p}-\frac{(z-1)}{\left(1-|\rho|^{2}\right)}|\rho|^{2} u u^{*}\right)^{-1}
\end{aligned}
$$

and

$$
\begin{aligned}
Q(z)= & \left(\left(I_{p}+\frac{(z-1)}{\left(1-|\rho|^{2}\right)} \bar{u} \bar{u}^{*}\right) R_{1}(z)-\frac{(z-1)}{\left(1-|\rho|^{2}\right)} \rho \bar{u} u^{*}\right) \\
& \left(\frac{(z-1)}{z\left(1-|\rho|^{2}\right)} \bar{\rho} u \bar{u}^{*} R_{1}(z)+I_{p}-\frac{(z-1)}{z\left(1-|\rho|^{2}\right)} u u^{*}\right)^{-1},
\end{aligned}
$$

which is precisely $Q=T_{\Theta_{\delta_{0}}}\left(R_{1}\right)$. Moreover, by Lemma 1 $R_{1}$ is symmetric which proves the result in this case.

Then, we assume that the interpolation data are of the form $\delta_{1}=(w, u, 0, \rho)$, so that (22) has the form

$$
Q(w) u=0,
$$

and that the associated Pick matrix

$$
\Lambda_{\delta_{1}}=\left[\begin{array}{cc}
\frac{1}{1-|w|^{2}} & \bar{\rho} \\
\rho & \frac{1}{1-|w|^{2}}
\end{array}\right]
$$

is positive definite. Consider the inner function $Q_{w}$ defined by

$$
Q_{w}\left(\beta_{w}(z)\right)=Q(z),
$$

where $\beta_{w}(z)$ is the Blaschke factor defined in (18). The matrix $Q_{w}$ is symmetric, has same McMillan degree as $Q(z)$ and satisfies the interpolation conditions

$$
Q_{w}(0) u=0, \quad u^{T} Q_{w}^{\prime}(0) u=\rho\left(1-|w|^{2}\right) \frac{1-w}{1-\bar{w}},
$$

associated with the interpolation data

$$
\delta_{1}^{\prime}=\left(0, u, 0, \rho\left(1-|w|^{2}\right) \frac{1-w}{1-\bar{w}}\right) .
$$

The Pick matrix associated with these data

$$
\Lambda_{\delta_{1}^{\prime}}=\left[\begin{array}{cc}
1 & \bar{\rho}\left(1-|w|^{2}\right) \frac{1-\bar{w}}{1-w} \\
\rho\left(1-|w|^{2}\right) \frac{1-w}{1-\bar{w}} & 1
\end{array}\right]
$$


is positive definite since $\Lambda_{\delta_{1}}$ is. The previous result thus applies to the matrix $Q_{w}$, so that

$$
Q_{w}=T_{\Theta_{\delta_{1}^{\prime}}}\left(R_{w}\right)
$$

for some symmetric inner function $R_{w}$ of McMillan degree $(n-2)$. It is then easily verified that

$$
\Theta_{\delta_{1}^{\prime}}\left(\beta_{w}(z)\right)=\Theta_{\delta_{1}}(z)
$$

so that

$$
Q(z)=Q_{w}\left(\beta_{w}(z)\right)=T_{\Theta_{\delta_{1}}}(R),
$$

in which $R(z)=R_{w}\left(\beta_{w}(z)\right)$ is symmetric and has degree $(n-2)$.

Now, we come to some general data $\delta=(w, u, v, \rho)$ for which $v$ is a nonzero vector. We first assume that $E=Q(w)$ is a strictly contractive matrix and we consider the function $\hat{Q}=T_{H(-E)}(Q)$

$=\left(I_{p}-E E^{*}\right)^{-1 / 2}(Q-E)\left(I_{p}-E^{*} Q\right)^{-1}\left(I_{p}-E^{*} E\right)^{1 / 2}$

where $H(E)$ is the Halmos extension (21) associated with the symmetric matrix $E$. This ensures that $\hat{Q}$ is symmetric. Moreover, we have that

$$
\hat{Q}^{\prime}(w)=\left(I-E E^{*}\right)^{-1 / 2} Q^{\prime}(w)\left(I-E^{*} E\right)^{-1 / 2},
$$

so that $\hat{Q}$ satisfies the set of interpolations conditions

$$
\hat{Q}(w) \hat{u}=0, \quad \hat{u}^{T} \hat{Q}^{\prime}(w) \hat{u}=\frac{\rho}{1-\|v\|^{2}},
$$

where $\hat{u}=\left(I-E^{*} E\right)^{1 / 2} \frac{u}{\sqrt{1-\|v\|^{2}}}$. The Pick matrix $\Lambda_{\hat{\delta}}$ associated with the interpolation data $\hat{\delta}=\left(w, \hat{u}, 0, \frac{\rho}{1-\|v\|^{2}}\right)$ is again strictly positive since $\Lambda_{\delta}$ is. Thus, by what precedes,

$$
\hat{Q}=T_{\Theta_{\hat{\delta}}}(\hat{R}),
$$

for some symmetric inner function $R$ of McMillan degree $(n-2)$. We then have (note that $H(E)^{-1}=H(-E)$ )

$$
Q=T_{H(E) \Theta_{\hat{\delta}} H(-E)}(R),
$$

in which $R=T_{H(E)}(\hat{R})$ is again symmetric of McMillan degree $(n-2)$. It remains to prove that

$$
H(E) \Theta_{\hat{\delta}} H(-E)=\Theta_{\delta} .
$$

This follows immediately from the fact that

$$
H(E)\left[\begin{array}{cc}
0 & -\overline{\hat{u}} \\
\hat{u} & 0
\end{array}\right]=\frac{1}{\sqrt{1-\|v\|^{2}}}\left[\begin{array}{cc}
v & -\bar{u} \\
u & -\bar{v}
\end{array}\right] .
$$

In the case where $Q(w)$ is not strictly contractive, we can write the Takagi's factorization ([9, Cor.4.4.4] for $Q(w)$

$$
Q(w)=U \operatorname{diag}\left(\sigma_{1}, \sigma_{2}, \ldots, \sigma_{n}\right) U^{T},
$$

where $U$ is a unitary matrix and the $\sigma_{k}$ 's are real nonnegative numbers of modulus less than or equal to one, since $Q(w)$ is contractive. Assume that $\left|\sigma_{1}\right|=\left|\sigma_{2}\right|=\cdots=\left|\sigma_{l}\right|=1$ while $\left|\sigma_{k}\right|<1$, for $k>l$, and choose

$$
E=U \operatorname{diag}\left(0, \ldots, 0, \sigma_{l+1}, \ldots, \sigma_{n}\right) U^{T} .
$$

It is not difficult to prove that $E u=v$ and the previous proof can be adapted to this case.

Remark 3: If both $v$ and $\rho$ are zero in the interpolation conditions (22), (23) and (24), the linear fractional representation $Q=T_{\Theta_{\delta}}(R)$ is a symmetric Potapov factorization

$Q(z)=\left(I_{p}+\left(\beta_{w}(z)-1\right) \bar{u} u^{T}\right) R(z)\left(I_{p}+\left(\beta_{w}(z)-1\right) u u^{*}\right)$.

The Schur algorithm: let $Q(z)$ be an inner function of McMillan degree $n \geq 2$. Then,

(i) we can find a set of interpolation data $\delta=(w, u, v, \rho)$ such that $Q(z)$ satisfies (22), (23), (24) and the Pick matrix $\Lambda_{\delta}$ given by (27) is positive definite.

Indeed, we can take for example a zero $w$ of $Q(z)$ and an associated direction $u$, so that

$$
Q(w) u=0
$$

Then, let $\rho=u^{T} Q^{\prime}(w) u$. By Schwartz lemma [12, chap.12] applied to the Schur analytic function $u^{T} Q(z) u$, we have

$$
|\rho| \leq \frac{1}{1-\|w\|^{2}}
$$

The equality is strict and thus the Pick matrix $\Lambda_{\delta}$ positive definite unless if $u^{T} Q(z) u=\xi \beta_{w}, \xi$ unit complex number. In this case, the matrix $Q(z)$ is of the form

$$
Q(z)=X \operatorname{diag}\left(\beta_{\omega}(z), Q_{1}(z)\right) X^{T},
$$

and it is not difficult to find a unit vector $u$ such that $u^{T} Q^{\prime}(\omega) u=\rho=0$ and $Q(\omega) u=v$, with $\|v\|<1$. The Pick matrix $\Lambda_{\delta}$ is again positive definite.

(ii) given a set of interpolation data $\delta$ such that the associated Pick matrix $\Lambda_{\delta}$ is positive definite, any matrix $Q$ satisfying (22), (23), (24) can be represented by a linear fractional transformation of the form

$$
Q=T_{\Theta_{\delta}}(R),
$$

for some symmetric inner function $R$ of McMillan degree $(n-2)$.

Repeating this process we construct a sequence of symmetric inner function

$$
Q_{n}=Q, Q_{n-2}, \ldots, Q_{n-2 i}, \ldots Q_{f},
$$

in which $Q_{n-2 i}$ has McMillan degree $(n-2 i)$. The Schur algorithm stops when the degree is 1 or 0 . If $n$ is odd, then $Q_{f}$ is a symmetric inner function of McMillan degree 1 while if $n$ is even, then $Q_{f}$ is a symmetric unitary matrix. These elementary symmetric inner functions were described in section III.

\section{SCHUR PARAMETERS FOR A SAW FILTER. NORMALIZATION ISSUES.}

We return to the example proposed in the introduction. Let $M_{n}(z)=Q_{n}(z) / z$ where $Q_{n}(z)$ is the acoustic matrix given by (2). It satisfies the interpolation condition (22) with

$$
w=0, \quad u=\left[\begin{array}{l}
0 \\
1
\end{array}\right], \quad v=\left[\begin{array}{c}
0 \\
-j r_{n}
\end{array}\right] .
$$


It can be verified that $u^{T} Q_{n}^{\prime}(0) u=0$, so that the associated Pick matrix $\Lambda=t_{n}^{2} I_{2}$ is clearly positive definite. The associated $J$-inner matrix can be computed as

$$
\Theta_{n}(z)=\left[\begin{array}{cccc}
1 & 0 & 0 & 0 \\
0 & \frac{z^{2}-r_{n}^{2}}{z t_{n}^{2}} & 0 & \frac{j r_{n}\left(z^{2}-1\right)}{z t_{n}^{2}} \\
0 & 0 & 1 & 0 \\
0 & \frac{j r_{n}\left(z^{2}-1\right)}{z t_{n}^{2}} & 0 & \frac{1-r_{n}^{2} z^{2}}{z t_{n}^{2}}
\end{array}\right] .
$$

We may also run two steps of the classical tangential Schur algorithm described in section II, with

$$
\begin{gathered}
w_{n}=0, \quad u_{n}=\left[\begin{array}{l}
0 \\
1
\end{array}\right], \quad v_{n}=\left[\begin{array}{l}
0 \\
0
\end{array}\right], \\
w_{n-1}=0, \quad u_{n-1}=\left[\begin{array}{l}
0 \\
1
\end{array}\right], \quad v_{n-1}=\left[\begin{array}{c}
0 \\
-j r_{n}
\end{array}\right] .
\end{gathered}
$$

We get

$$
M_{n}=T_{\Phi_{n}}\left(M_{n-1}\right)
$$

with

$$
\Phi_{n}(z)=\left[\begin{array}{cccc}
1 & 0 & 0 & 0 \\
0 & \frac{z}{t_{n}} & 0 & \frac{-j r_{n}}{z t_{n}} \\
0 & 0 & 1 & 0 \\
0 & \frac{j r_{n} z}{t_{n}} & 0 & \frac{1}{z t_{n}}
\end{array}\right]
$$

Note that this matrix doesn't satisfy the condition $\Phi_{n}(1)=$ $I_{2 p}$ that was imposed to the $J$-inner functions $\Theta_{\delta}$ in the symmetric Schur algorithm. The matrix $\Phi_{n}(1)$ is in fact equal to $H\left(v u^{*}\right)$. Note that, in this particular case,

$$
v u^{*}=\left[\begin{array}{cc}
0 & 0 \\
0 & -j r_{n}
\end{array}\right],
$$

is symmetric, so that by Lemma 4 and Lemma 1 the linear fractional transformation $T_{H\left(v u^{*}\right)}$ preserves symmetry. Moreover, we have that

$$
\Phi_{n}=\Theta_{n} \Phi_{n}(1)
$$

It appears that the sequence of acoustic matrices $M_{n}$ corresponds to a symmetric Schur algorithm in which the $J$-inner function $\Theta_{\delta} H\left(v u^{*}\right)$ has been chosen instead of $\Theta_{\delta}$.

This leads us to some considerations on the choice of the $J$-inner function in a Schur algorithm. Concerning the tangential Schur algorithm described in section II, we have already mentioned that several choices have been made in the literature (see remark 1) and in particular, that of [10] which allows for a nice circuit interpretation, and that of [8] which leads to a recursive construction of balanced realizations. In the context of SAW filters, the Schur algorithm associated with the matrices $\Phi_{n}$ has a nice physical interpretation: it gives the sequence of acoustic matrices $M_{n}$ corresponding to the first $n$-cells of the filter. Moreover, it can be proved that it allows for a recursive construction of balanced realizations as described in [8]. This is not very surprising since, when the interpolation point $w$ is zero, the choices in [10] and [8] for the matrix $\Theta$ agree. In general, the matrix $v u^{*}$ is not symmetric. It is symmetric only if $v=\lambda \bar{u}$ for some complex number $\lambda$. This raises the question of the choice of a symmetric contractive matrix $E$, such that the Schur algorithm associated with $\Theta_{\delta} H(E)$ allows for a recursive construction of balanced realizations.

\section{REFERENCES}

[1] D. Alpay, L. Baratchart, A. Gombani, On the differential structure of matrix-valued rational inner functions, Operator Theory : Advances and Applications, 73 (1994), pp. 30-66.

[2] J. BALL, I. GohBerg, L. Rodman, Interpolation of rational matrix functions, Operator Theory: Advances and Applications Vol.45, Birkhäuser, 1990.

[3] L. Baratchart, A. Gombani, M. Olivi, Parameter determination for surface acoustic wave filters, in Proceedings of the CDC, Sydney, Australia, 2000.

[4] H. DYM, J-contractive matrix functions, reproducing kernel spaces and interpolation, CBMS lecture notes, No. 71, American Mathematical Society, Rhode Island, 1989.

[5] P. Fulcheri AND M. Olivi, Matrix Rational $H^{2}$-Approximation: a Gradient Algorithm Based on Schur Analysis, SIAM J. Contr. and Opt., 36 (1998), pp. 2103-2127.

[6] P.A. Fuhrmann, On symmetric rational matrix functions, Lin. Alg.Appl., Special issue on Linear Control Theory, (1983), 167-250.

[7] U. Helmke AND P.A. Funrmann, Tangent spaces of rational functions, Lin. Alg. Appl., (1998), vol. 271, 1-40.

[8] B. Hanzon, M. Olivi, R.L.M. Peeters, Balanced realizations of discrete-time stable all-pass systems and the tangential Schur algorithm, INRIA research report No. 5111, 2004, to appear in Linear Algebra and Applications.

[9] R. A. Horn and C. R. Johnson, Matrix Analysis, Cambridge University Press, Cambridge, UK, 1985.

[10] H. KIMURA, Directional interpolation approach to $H^{\infty}$-optimization and robust stabilization, IEEE transactions on automatic control, Vol. AC-32 (1987), No. 12, pp. 1085-1093.

[11] V.P. PoTAPOV, The multiplicative structure of J-contractive matrix functions, Amer. Math. Soc. Transl. (2) 15 (1960), pp. 131-243.

[12] W. RUDIN Real and complex analysis, McGraw-Hill (1966). 